\title{
Structural diagrams and basic design relationships for the calculation of road safety indicators
}

\author{
Bazhanov Anatoly Pavlovich \\ Penza State University of Architecture and Construction \\ Penza, Russia \\ bajan_p@mail.ru
}

\begin{abstract}
- main tasks of research and maintenance of reliability at the present stage of designing and construction of highways are stated. The sequence of the analysis of structural reliability schemes of non-rigid road clothes under conditions of impact on them of normal and limiting values of transport operational loads and weather climatic conditions - is considered. During the analysis of structural reliability schemes, the quantitative characteristics of the reliability of layers of the road structure are determined on the basis of the representation of the physical model of their failure, which includes a complete group of events (failures) of the layers of the road structure and the road structure in general.

In this case, the model of failure of each layer is represented by a small number of determining factors ("limiting" parameters, such as the modulus of elasticity, the smoothness of the road surface, rutting, cracking, etc.) allowing their quantitative measurement or calculation according to the corresponding deterministic relationships. These correlations are usually found from the theory of designing the corresponding typical road projects or from the results of specially set experiments
\end{abstract}

Keywords - quantitative methods of reliability research, structural reliability schemes, layers of road structure, roadway cover defects, determining factors, "limit" parameters, normal distribution law of random variables, coefficient of correlation of random variables, lower confidence limit of reliability assessment.

\section{INTRODUCTION}

The basis of research and ensuring reliability at the current stage of construction of roads in their design is the following tasks:

- improving the requirements for the reliability of highways and selection of the main ways to achieve them;

- ensuring the required level of reliability in the framework of the design concepts of highways, taking into account the limitations associated with the modern scientific and technological level and the resources allocated for their construction;

- carrying out an analysis of estimates of indicators of road reliability with the selected in the design process technical solutions. The resulting estimates of reliability indicators (and, first of all, reliability factors) are used when comparing the variants of various pre-design and design solutions that most satisfy the reliability requirements specified in the design documentation, as well as to justify subsequent decisions;

- distribution of the main available types of resources (cash, labor, material, temporary) to ensure the reliability of the designed roads, implemented at all periods and stages of their life cycle. These include the task of justifying the requirements for reliability, the choice of effective means of monitoring the quality of highways, maintaining reliability during operation, etc.

\section{MATERIALS AND METHODS OF RESEARCH}

All of the mentioned tasks can now be successfully solved thanks to the use of various quantitative methods of reliability research based on the modeling of pre-design and design solutions based on the use of modern high-duty computer engineering.

For the quantitative study and assessment of the reliability of highways, it is necessary to do analysis of structural schemes of their reliability [1]. These schemes are constructed in the form of successive parallel structures, which include all pavement layer of the motor road, the fault of which can lead to failures in its operation.

In the course of the analysis of structural reliability schemes, the consequences of the destruction of the road structure, which is an engineering structure consisting of pavement and the upper part of the roadbed within the working layer, are investigated.

Let us consider the sequence of analysis, for the example, of the recommended designs of non-rigid pavements in conditions of impact on them of an intensive cargo transport stream for highways of the I-II categories (Figure 1) [2].

In accordance with the presented figure, the construction of road clothes consists of: layers of the coating, the base and additional layers of the base. The functional and constructive purpose of these layers is to ensure the coupling of the coating, its resistance to abrasion, the provision of temperature cracking, load-bearing capacity, resistance to wheel track rutting and fatigue cracking.

In the process of constructing structural reliability schemes, the conditions of operation of a highway under normal (calculated) and limiting values of its loads and types of defects are investigated. Limit values of loads and the size 
of defects lead to the destruction of each of the layers of the road structure, especially regarding the analysis of the causes and consequences of the destruction of the upper and lower of these layers, the state of which is recognized as the limiting one. Therefore, if the road load limits are exceeded, its operation is not allowed, difficult or impractical [3].
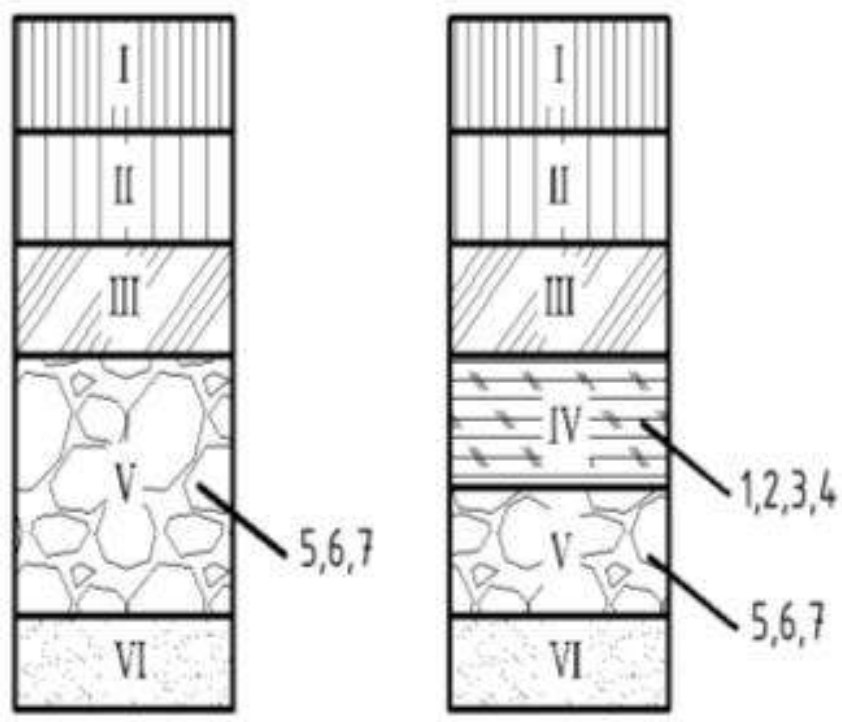

Fig. 1. Examples of recommended pavement designs

I - upper layer of coating: hot dense (high-density)m / $\mathrm{z}$ asphalt concrete, PMA; II - the lowest layer of the coating: hot dense (porous) c / o or m / s asphalt concrete; III - the top layer of the base: hot porous (dense) asphalt concrete ; IV - base (1- mixtures of rubble-gravel-sand, treated with inorganic

bonding materials, 2 - rubble treated with viscous bitumen or bitumen emulsion, 3 - organomineral mixtures, 4 - rubble-gravel-sand mixtures treated with ash or slag binder, $\mathrm{V}$ - base (5 - rubble-gravel-sand mixes with continuous granulometry of grade C4-C6, 6 - fractionated rubble 40-70 (70-

120) $\mathrm{mm}$ jammed with rubble , 7 - soils treated with inorganic bonders VI - an additional layer of the base (mixtures C3-C6 and C9-C11, sands).

Based on the results of analysis of structural reliability diagrams, the value of the level of reliability of the road structure should be assigned depending on the total number of applications of the design load and the quantity of permissible standard wear of the upper layer of the coating caused by its defects that most significantly affect the reliability of the road structure (Table 1) [4, 5].

The main quantitative characteristics of the reliability of the road structure in general or any of its layers in the hierarchical structure are:

- $\quad$ probability of $\mathrm{P}$ failure-free operation;

- $\quad$ the mean square deviation $\sigma$ of the probability $\mathrm{P}$

In practice, instead of probability $\mathrm{P}$, it is determined by its point estimate $P$ and interval estimation in the form of lower confidence limit $\underline{\mathrm{P}}$ with some given confidence level $\gamma$. The values of $\mathrm{P}$ and $\underline{\mathrm{P}}$ together characterize the accuracy of estimating the reliability of the road structure. A measure of the accuracy of the estimate $\mathrm{P}$ of the probability $\underline{\mathrm{P}}$ can also be estimate $S_{P}$ of mean square deviation $\sigma_{P}$
TABLE 1. TYPES OF DEFECTS IN COVERING THE ROADWAY OF A ROAD

\begin{tabular}{|c|c|}
\hline Types of defects & Describing of defects \\
\hline Pot holes & $\begin{array}{l}\text { The destruction of the } \\
\text { coating in the form of } \\
\text { depressions of various shapes } \\
\text { with sharply defined edges } \\
\text { (more than } 3 \mathrm{~cm} \text { deep and an } \\
\text { area of more than } 200 \mathrm{~cm} \text { ) }\end{array}$ \\
\hline Bracks & $\begin{array}{l}\text { Destruction of pavement for } \\
\text { its entire thickness with a sharp } \\
\text { distortion of the transverse } \\
\text { profile, accompanied by a grid } \\
\text { of cracks }\end{array}$ \\
\hline $\begin{array}{l}\text { Untreated sections of the } \\
\text { roadway with bleeding of } \\
\text { bonding material }\end{array}$ & $\begin{array}{l}\text { Surplus of bonding material } \\
\text { on the surface of the coating } \\
\text { with a change in its texture and } \\
\text { color, area of more than } 1 \mathrm{~m}\end{array}$ \\
\hline Cracks & $\begin{array}{l}\text { Violation of the integrity of } \\
\text { the coating without removing } \\
\text { the material with the formation } \\
\text { of narrow slits. Cracks of } \\
\text { arbitrary outline and location } \\
\text { with a width of opening more } \\
\text { than } 3 \mathrm{~mm} \text { on the roadway }\end{array}$ \\
\hline Temperature cracks & \begin{tabular}{l}
\multicolumn{1}{c}{ Cracks caused by } \\
temperature fluctuations and \\
stress concentrations in the \\
coating layers
\end{tabular} \\
\hline $\begin{array}{l}\text { The grid of cracks along } \\
\text { the bands of rolling }\end{array}$ & $\begin{array}{l}\text { The destruction of the } \\
\text { coating without significant } \\
\text { distortion of the profile and } \\
\text { accompanying with small } \\
\text { longitudinal and transverse an } \\
\text { arbitrary outline cracks, } \\
\text { characteristic of insufficient } \\
\text { pavement strength }\end{array}$ \\
\hline $\begin{array}{l}\text { Destroyed and not filled } \\
\text { with mastic deformation } \\
\text { seams on cement concrete } \\
\text { coating }\end{array}$ & \begin{tabular}{l}
\multicolumn{1}{c}{ Damage of joints and } \\
materials near them, presence of \\
debris in damaged joints and \\
inclusions of armouring \\
elements
\end{tabular} \\
\hline
\end{tabular}

When calculating the reliability, the point estimate $\mathrm{P}$ of the probability $\mathrm{P}$ of the fail-safe operation of the road structure, which is a successive chain of its layers (Fig. 2), assuming independence of failures in the operation of these layers, is calculated from the formula [6]:

$$
P={ }_{i=1}^{N} P_{i}(1)
$$

where $P_{i}$ is the estimate of probability $\mathrm{P}$ of the fail-safe operation of the $i$-th layer of the road structure $(i=\overline{1, N}, N$ number of layers in the structural reliability schemes and undesirable events that are related to the road structure as a whole). 


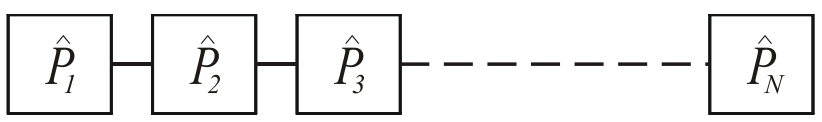

Fig. 2. Standard structural reliability scheme as a successive chain of structural layers of the road structure.

Lowest confidence limit $\underline{\mathrm{P} \gamma}$ of the fail-safe operation estimate of the road structure with significance level $1-\gamma$ (where $\gamma$ is the confidence level) and the estimate of mean square deviation $S_{P}$ of point estimate $P$ are determined by the formulas [6]:

$$
\underline{P}_{\gamma}=P 1-\overline{{ }_{i=1}^{N}\left(1-\underline{P}_{i} / P_{i}\right)^{2}}
$$

where $P_{i}$ is the lowest confidence limit of the $P_{i}$ estimate of the fail-safe operation of the i-th layer of the road structure:

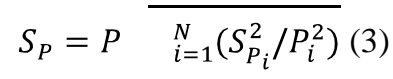

In determining the quantitative characteristics of the reliability of the layers of a road structure, a physical model of their failure is established, which includes a complete group of events leading to the failure of the layers of the road structure and the road structure as a whole. In this case, the failure model of each layer is described by a small number of determining factors ("limiting" parameters) that allow their quantitative measurement or calculation according to the corresponding deterministic relations. These ratios are usually known from the theory of designing the corresponding standard road projects or from the results of specially set experiments.

In the case of the performance characteristics of a layer of a road structure (or a road structure as a whole) by one defining parameter $\mathrm{x}$, the condition of its fail-safe is defined as:

$$
p x_{\text {lim }}-x_{n}=u>0 \text { (4) }
$$

where $x_{\text {lim }}$ is the limiting value of the determining parameter $\mathrm{x}$, at which the layer (and, consequently, the road structure as a whole) uniquely refuses to work; $x_{\mathrm{n}}$ is the normative value of the determining parameter $\mathrm{x}$, at which the layer of the road structure functions faultlessly.

So, for example, for road construction of non-rigid road clothes under conditions of influence of an intensive cargo transport stream for highways of I-II categories as defining parameters $\mathrm{x}$ can be accepted: $x_{1}$ is the modulus of elasticity, $x_{2}$ is the smoothness of the pavement, $x_{3}$ is the wheel tracking, $x_{4}$ is the cracking etc. In this case, $x_{\lim 1}$ will be the limiting values of the modulus of elasticity, $x_{\lim 2}$ - smoothness, $x_{\lim 3}$ wheel tracking, $x_{\text {lim } 4}$ - cracking, which unambiguously do not provide trouble-free functioning of the road structure.

And for $x_{\mathrm{n} 1}, x_{\mathrm{n} 2}, x_{\mathrm{n} 3}, x_{\mathrm{n} 4}$ such normative values of modulus of elasticity, smoothness and wheel tracking, which ensure trouble-free functioning of the road structure with a probability not less than the value specified in the design documentation.

For the case where the reliability of a layer or road structure as a whole is characterized by one defining parameter $x$ being a random variable, the probability of its failure-free operation is determined by the relation:

$$
P=p x_{\text {lim }}-x_{n}=u>0={ }_{0}^{h} \varphi(u) d u(5)
$$

where $\varphi(u)$ is the composition of the distribution laws (probability densities) of the random variables $x_{\text {lim }}$ and $x_{\mathrm{n}} ; h$ is a certain limit, calculated depending on the distribution law of the random variable $u$.

Under the normal distribution law of the random variables $x_{\text {lim }}$ and $x_{n}$, the estimate $P$ of the probability $\mathrm{P}$ can be calculated from the formula by A.R. Rzhanitsin [6]:

$$
P=F(h)(6)
$$

where $F h=1 / 2 \pi \stackrel{h}{h} \exp \left(-x^{2} / 2\right) d x$ - the tabulation in [7] the function of the standardised normal distribution;

$$
\begin{gathered}
h=\left(\bar{x}_{l i m}-\bar{x}_{n}\right) / 1 /\left(S_{x_{l i m}}^{2}+S_{x_{n}}^{2}-2 S_{x_{l i m}} \cdot S_{x_{n} .} .\right. \\
\left.r_{x_{l i m}, x_{n}}\right)^{1 / 2}(7)
\end{gathered}
$$

Here: $\bar{x}_{\text {lim }}, \bar{x}_{\mathrm{n}}, S_{x_{\text {lim }},}, S_{x_{\mathrm{n}}}, r_{x_{\text {lim }}, x_{\mathrm{n}}}$ are estimates of mean values, mean square deviations and the pair coefficient of correlation of the random variables $x_{\text {lim }}$ and $x_{\mathrm{n}}$. These quantities at different stages of the design phase are determined by analytical methods using the calculated ratios describing the values of $x_{\text {lim }}$ and $x_{\mathrm{n}}$, and using the statistical modeling method using the following formulas:

$$
\begin{aligned}
& \bar{x}_{\text {lim }}=\frac{1}{n_{1}} \quad n_{i=1}^{n_{1}} x_{\lim _{i}}, \bar{x}_{n}=\frac{1}{n_{2}} \quad n_{i=1}^{n_{2}} x_{n_{i}}(8) \\
& S_{x_{\text {lim. }}}=\frac{1}{n_{1}-1}{ }_{i=1}^{n_{1}}\left(x_{\lim _{i}}-\bar{x}_{\text {lim }}\right)^{2}{ }^{1 / 2} \text {, } \\
& S_{x_{n} .}=\frac{1}{n_{2}-1} \quad n_{i=1}\left(x_{n_{i}}-\bar{x}_{n}\right)^{2} \\
& r_{x_{l i m}, x_{n}}=\frac{1}{n s_{x_{l i m}} \cdot s_{x_{n}}}{ }_{i=1}^{n}\left(x_{i l i m}-\bar{x}_{l i m}\right)\left(x_{i n}-\bar{x}_{i n}\right),
\end{aligned}
$$

where $n_{1}, n_{2}, n$ are the numbers of realizations of the random variables $x_{l i m}$ and $x_{\mathrm{n}}$ ), by which the statistics values $\bar{x}_{\text {lim }}, \bar{x}_{\mathrm{n}}, S_{x_{\text {lim }},}, S_{x_{\mathrm{n}}}, r_{x_{\text {lim }}, x_{\mathrm{n}}}$ will be assigned.

For the case where the reliability of a road structure is determined simultaneously by several defining parameters (for example, for a road structure, this can be both the elastic modulus- $x_{1}$, and the smoothness $-x_{2}$, etc.), a multidimensional failure model is used. Then the failure-free condition is represented as:

$$
\begin{gathered}
p x_{\lim 1}-x_{n 1}=u_{1}>0 \\
p x_{\lim 2}-x_{n 2}=u_{2}>0 . \\
\ldots \ldots \ldots \ldots \ldots \ldots \ldots \ldots \ldots \\
p x_{\lim N}-x_{n N}=u_{N}>0
\end{gathered}
$$

where $\mathrm{N}$ is the number of defining parameters $x_{j}(j=$ $\overline{1, N}$ ), control points, fail-safe conditions, calculation cases, etc., which are used in constructing models of failures of the road structure as a whole, its layers and individual structural elements. 
Based on the conditions of non-failure of type (11), the component of reliability in the form of a possible trouble-free functioning of the road structure (layer) in this case can be calculated according to the following relationship:

$$
P=\begin{array}{lllll}
h_{1} & h_{2} & \ldots & h_{N} \\
0 & 0 & \cdots & 0
\end{array} u_{1}, u_{2}, \ldots u_{N}, d u_{1} d u_{2} \ldots d u_{N}
$$

For an arbitrary distribution law of the integrand (12), calculating the probability $\mathrm{P}$ is very problematic, especially for a sufficiently large multiplicity of the integral ( $N>10)$. For a small multiplicity of the integral (12) (of the order of ones), it is calculated by the method of statistical modeling, using the Kendall series [8] or the Iyudu solutions [9]. One can also use the decomposition, which can be performed in the multidimensional Gramma-Charlier series

For the road structure, the defining parameters $x_{j}(j=$ $\overline{1, N}) ; \mathrm{N}$ is the number of determining parameters) in many practical cases are considered to be distributed according to the normal law. In this case, for the conditions of no-failure type (11), the point estimate $\mathrm{P}$, the probability $\mathrm{P}$ can be calculated from the approximate relation by R.S. Sudakov (with an error of the order of $2-8 \%$ of the value of 1-P) of the following form [6]:

$$
\begin{gathered}
P={ }_{j=1}^{N} P_{j}+P_{j \min }-{ }_{j=1}^{N} P_{j} K_{N}= \\
={ }_{j=1}^{N} F\left(h_{j}\right)+F\left(h_{j}\right)_{\min }-{ }_{j=1}^{N} F\left(h_{j}\right) K_{N},(13)
\end{gathered}
$$

where $F\left(h_{j}\right)_{\min }$ - the minimum of the estimates $F\left(h_{j}\right)$,

$$
K_{N}=\frac{2}{\pi c} \underset{i<j}{\arcsin r_{i j}}
$$

$r_{i j}$-estimation of the pair correlation coefficient of the random variables $x_{п р}$ and $x_{\mathrm{H}}$ (between the parameters $x_{j}$ ); the subscript $" i<j$ " under the summation sign denotes the number of all possible pair combinations under conditions of nonfailure (11), the number of such conditions is $\mathrm{c}=\mathrm{N}(\mathrm{N}-1) / 2$.

It was shown in the paper [10] that for small numbers $\mathrm{N}$ (of the order of ones and probabilities $P_{j} \geq 0,999$, the value of the coefficient $K_{N}$ is close to 0.5 . In this case, the relation (1.18) is simplified and has the form:

$$
P={ }_{j=1}^{N} P_{j}+\frac{1}{2}\left(P_{j_{\text {min }}}-{ }_{j=1}^{N} P_{j}\right)
$$

\section{RESULTS OF RESEARCH}

In practical calculations for the road construction, the values of the estimates $P_{j}<0,999$. In such cases, in accordance with the data given in the paper [11], the random variables $x_{\text {пр }}$ and $x_{\mathrm{H}}$ can be considered almost uncorrelated. Consequently, estimates $h_{j}$ parameters $h_{j}$ can be calculated from simpler formula of the form:

$$
h_{j}=\bar{x}_{l i m}-\bar{x}_{n} \quad \overline{S_{x_{l i m}}^{2}+S_{x_{n}}^{2}}
$$

The lowest confidence limit P P_ $\gamma$ of estimate (13) in the general case can be calculated from the formula [6]
$\underline{P}_{\gamma}=$

$P 1-$

$$
\overline{\left(1-\frac{P_{j \min }}{P_{j \min }}\right)^{2}+\left(1-K_{N}^{2}\right)\left({\left.\underset{j=1}{N} P_{j} / P\right)}_{j=2}^{N}\left(1-\underline{P}_{j} / P_{j}\right)^{2}\right.} .
$$

In the last formula (16), the estimate of $\mathrm{P}$ is calculated from the relations (13), estimates $P_{j}(j=\overline{1, N})$ - using formula (6) using formulas (15), and the lower confidence limits $\underline{P}_{j}$ of estimates $\mathbb{E}_{j}$ can be calculated from the well-known $D$. Cowden series [12]:

$$
\underline{P}_{j}=F \quad h_{j}-\frac{h_{1-\alpha}}{\bar{h}} \overline{1+h^{2} / 2},
$$

where the estimates of $h_{j}$ are calculated by formula (15); The quantile values $h_{1-\alpha}$ are found from the statistical tables [7], depending on the confidence probability $\gamma=1-\alpha$. For practical calculations, $\alpha$ is most often taken to be 0,05 . At this value $h_{1-\alpha}=1,645$. If necessary, i.e. for other values of the significance levels $\alpha$ one can use the same most known tables [7].

\section{References}

[1] Method of structural reliability scheme, GOST R 51901.14-2005. Moscow: Standardinform, 2005

[2] ODM 218.056-2015 Methodical recommendations on the design of nonrigid road clothes in conditions of the impact of intensive freight traffic flow (for motor roads of I-II categories), Enter. 2001-07-01, Moscow: Dorinzhservis LLC, 2015.

[3] GOST R 54257-2010 Reliability of building structures and foundations. Basic provisions and requirements, Moscow: Standartinform, 2011.

[4] GOST 33180-2014 Public roads. Requirements for the level of summer content, M .: Standardinform, 2016. - $18 \mathrm{p}$.

[5] A.P Bazhanov, E.S. Saksonova, "The method of carrying out heuristic procedures in the selection and justification of the composition of the determining indicators of the quality of coatings affecting the indicators of road safety. Preparation and interview of experts", Transport construction № 2, pp. 1-12, Moscow: Transstroiizdat, 2018.

[6] A.P. Bazhanov, "The main provisions of the general methodology for assessing the mechanical component of the reliability of sensor equipment for rocket and space technology at the design stages", Reliability and quality control. The supplement to the journal Standards and Quality, № 5, pp. 18-26, 1997.

[7] L.N. Bolshev, N.V. Smirnov "Tables of mathematical statistics", Science, 1983.

[8] M. Kendall, A. Stewart, "The theory of distributions" Science, 1966, 588.

[9] K.A. Iyudu, "Optimisation of automation devices by reliability criterion", Soviet radio, 1962, 194.

[10] A.A. Zolotov, M.I. Titov, "Ensuring the reliability of transport vehicles of space systems", Mechanical Engineering, 1988, 216.

[11] N.F. Gusev, "Accounting for correlation relationships in calculating the reliability of mechanical devices and aggregates", Reliability and quality control. The supplement to the journal, Standards and Quality, №7, pp 27-29, 1969.

[12] D. Cowden, "Statistical methods of quality control", Fizmatgiz, 1961, 623. 